\title{
Amperometric Flow-Injection Biosensor System for the Simultaneous Determination of Urea and Creatinine
}

\author{
Chang-Sheng RuI*, Kenji Sonomoto** and Yasuhiko Kato*十 \\ *Department of Applied Chemistry, Faculty of Engineering, Kyushu Institute of Technology, \\ Kitakyushu 804, Japan \\ ** Department of Biochemical Engineering and Science, Faculty of Computer Science and System Engineering, \\ Kyushu Institute of Technology, Iizuka 820, Japan
}

\begin{abstract}
A flow-injection biosensor system was developed for the simultaneous assay of urea and creatinine, with a single injection and one detector. The amperometric detection of urea or creatinine was based on coupled reactions of three sequentially aligned enzyme reactors, urease or creatinine deiminase, glutamate dehydrogenase and glutamate oxidase. Ammonia produced by the enzymatic hydrolysis of urea or creatinine was converted to glutamate, and the oxygen consumption due to the oxidation of glutamate by glutamate oxidase was detected with an oxygen electrode. A split and confluence of the flow stream between the injector and the glutamate dehydrogenase reactor resulted in a two-channel system. Doublepeak recording was achieved by putting a delay coil at one of the two channels. The system gave linear calibration curves over a range of $0.1-5.0 \mathrm{mM}$ for both urea and creatinine. The assay procedure is simple and one run can be completed within $3 \mathrm{~min}$. The system was reproducible within $5 \%$ of the relative standard deviation.
\end{abstract}

Keywords Biosensor, amperometric detection, urea, creatinine, split and confluence of flow, multi-functionalization

Numerous biosensors based on different principles have been developed. A biosensor comprises a molecular recognition system in physical and functional contact with a transducer. Together with the abovementioned two components, for example in a biosensor based on flow-injection analysis (FIA), surrounding systems for propulsion, injection, data processing and temperature control etc. are also involved. The most essential analyte recognition part takes only a very small space. The cost of a biosensor is mainly due to the detection and surrounding systems. The development of multi-functional biosensors that simultaneously determine several compounds with a single set of surrounding systems, therefore, may provide a promising way to increase the efficiency of equipment and to reduce assay costs.

Few studies have been reported concerning the multifunctionalization of biosensors. An enzyme sensor used to determine three compounds for estimating fish freshness was developed ${ }^{1}$, based on the separation by column chromatography and sequential elution of analytes. Though the system was successful, the principle of separation was specific to those compounds and the assay procedure was complicated and timeconsuming (more than $15 \mathrm{~min}$ for a run). Systems using plural detectors, such as in the simultaneous determination of two transaminases ${ }^{2}$, are not considered to be

$\dagger$ To whom correspondence should be addressed. multi-functional, since the information level is not enhanced compared to conventional systems with regard to the detector. ${ }^{3}$

We propose in this study a bifunctional FIA biosensor system for the simultaneous determination of urea and creatinine, with a single sample injection and one detector. The inclusion of splitting and confluence points, between which the sample zone is split, results in a two-channel FIA system. Multiple-peak recording is obtained by setting a delay coil at one of the two channels.

Urea and creatinine are very important nitrogen compounds of analytical interest; studies concerning biosensors for their determination have been widely reported. Most of them are based on the direct detection of ammonia produced by the hydrolysis of urea catalyzed by urease, or of creatinine catalyzed by creatinine deiminase (CRDI), using an ammonia gas or ammonia ion electrode. ${ }^{4}$ These potentiometric detectors are, however, known to suffer many types of interference, such as amines or potassium ions. Thus, amperometric microbial sensors using nitrifying bacteria have been developed..$^{5-7}$ The long assay time and low sensitivity, however, have limited their applications. In this study the amperometric detection of ammonia was carried out with a two enzyme system (glutamate dehydrogenase (GLDH)/glutamate oxidase (GLOD)) which has been used in the chemiluminometric assay of ammonia ${ }^{8}$, based on the following principle: 


$$
\begin{array}{r}
\underset{\text { 2-Oxoglutarate }+}{\mathrm{NH}_{4}{ }^{+}+\mathrm{NAD}(\mathrm{P}) \mathrm{H} \stackrel{\mathrm{GLDH}}{\longrightarrow}} \\
\text { L-glutamate }+\mathrm{NAD}(\mathrm{P})^{+}+\mathrm{H}_{2} \mathrm{O}, \\
\text { L-Glutamate }+ \\
+\mathrm{O}_{2}+\mathrm{H}_{2} \mathrm{O} \stackrel{\text { GLOD }}{\longrightarrow} \\
\text { 2-oxoglutarate }+\mathrm{NH}_{4}{ }^{+}+\mathrm{H}_{2} \mathrm{O}_{2} .
\end{array}
$$

The consumption of oxygen is finally detected with an oxygen electrode.

\section{Experimental}

\section{Materials}

Urease (EC 3.5.1.5) from Thermophilic Bacillus sp. was purchased from Wako Pure Chemical Industries (Osaka, Japan); CRDI (EC 3.5.4.21) was from Sigma (St. Louis, MO, USA); GLDH (EC 1.4.1.3) from beef liver and NADH were from Oriental Yeast (Osaka, Japan); GLOD (EC 1.4.3.11) from Streptomyces sp. was from Yamasa Shoyu (Chosi, Japan). Controlled Pore Glass (CPG, 120 - 200 mesh, $500 \AA$ pore size), propylamineCPG and succinate-CPG with the same size were obtained from Pierce Chemical Co. (Rockford, IL, USA). All other chemicals were commercially available and of the highest grade.

\section{Reagents}

Standard solutions and buffers were prepared with doubly-distilled water. The working solution to run the FIA systems was a $67 \mathrm{mM}$ potassium-sodium phosphate buffer (pH 8.0) containing $0.1 \mathrm{mM}$ EDTA, $5 \mathrm{mM} 2-$ oxoglutarate and $0.3 \mathrm{mM}$ NADH. Standard solutions of urea, creatinine and ammonia (as sulfate) were diluted with distilled water from the corresponding stock solutions, which were stored at $4^{\circ} \mathrm{C}$ and refreshed every week.

\section{Preparation of immobilized enzymes}

Urease, CRDI and GLOD were separately immobilized on propylamine-CPG pretreated with glutaraldehyde according to a method of Weetall. 9 GLDH was immobilized on succinate-CPG activated with 1-ethyl-3(3-dimethylaminopropyl)carbodiimide (EDC). ${ }^{10}$ For use in the FIA systems, immobilized enzymes were packed into glass tubes (i.d. $3.4 \mathrm{~mm}$ ). One centimeter of the tube corresponded to $36 \mathrm{mg}$ of dry CPG. When not used, the enzyme preparations were stored at $4^{\circ} \mathrm{C}$.

\section{Enzyme assay}

The assay of native GLDH was carried out using a procedure provided by the manufacturer. Native urease and CRDI were assayed using an NADH/GLDH coupled method. ${ }^{11}$ The activity of native GLDH was determined by an $o$-dianisidine/peroxidase method similar to the 4-aminoantipyrine/peroxidase assay described by Bohmer et al. ${ }^{12}$

Continuous flow through method was used for the assay of immobilized enzymes. First, the enzyme preparation (dry weight, $10-20 \mathrm{mg}$ ) was packed into a glass tube (i.d. $2.4 \mathrm{~mm}, 1 \mathrm{~cm}$ corresponds to $18 \mathrm{mg}$ dry beads). Then, the reaction mixture containing pertinent substrate(s) (and cofactors) was allowed to flow through the enzyme reactor. The effluent was sampled so as to immediately determine the concentration of ammonia for urease and CRDI, of NADH for GLDH, or of hydrogen peroxide for GLOD. The measured values of the activities increased with increasing flow rate, but remained constant when the flow rate was larger than a certain value, which depended on the total enzyme activity used in the assay. Therefore, the assay for each enzyme was performed at a flow rate somewhat larger than this critical point $(5-12 \mathrm{ml} / \mathrm{min})$. Regarding the $\mathrm{pH}$ dependence and kinetic assay, one column was used for each set of reaction mixtures. Essentially no activity loss was observed during the assay procedure. All of the experiments were performed at $25^{\circ} \mathrm{C}$.

\section{Flow-injection systems}

Figure 1 shows schematic diagrams of a single-channel FIA system used for the determination of one component (A), and a two-channel system for the simultaneous assay of urea and creatinine (B). Both systems involved a peristaltic pump (Atto Co., Tokyo, Japan), an injection port, enzyme reactors, a galvanic oxygen electrode equipped with a flow cell (Able Co., Tokyo), a

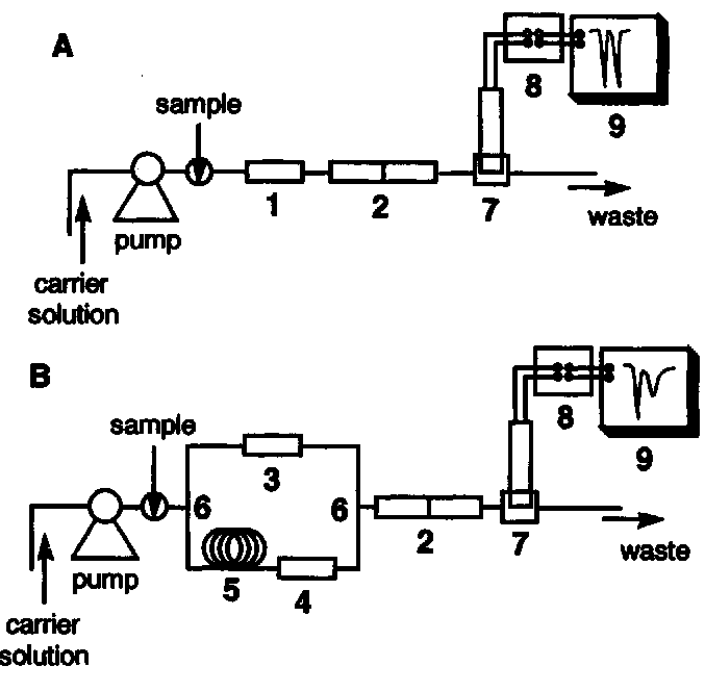

Fig. 1 Schematic diagram of the flow-injection systems for the amperometric assay of creatinine and urea: $A$, single channel system; B, two channel system. Enzyme reactors and the lengths (mm): 1, CRDI or urease (21); 2, GLDH/ GLOD (31/28); 3, CRDI (21); 4, urease (17). The inner diameter of all enzyme reactors was $3.4 \mathrm{~mm}$. 5, delay coil, i.d. $1.0 \mathrm{~mm}, 1.2 \mathrm{~m}$ long; 6 , T-connector; 7, oxygen electrode and flow cell; 8 , potentiostat; 9, recorder. The enzyme reactors and oxygen electrode were kept at $25^{\circ} \mathrm{C}$ with a thermostat. Samples were injected with a microsyringe and the injection volume was 10 or $50 \mu \mathrm{l}$. Flow rate: $1.6 \mathrm{ml} / \mathrm{min}$ for both systems. Working solution: $67 \mathrm{mM}$ phosphate buffer ( $\mathrm{pH}$ 8.0) containing $0.1 \mathrm{mM}$ EDTA, $0.3 \mathrm{mM} \mathrm{NADH}$ and $5 \mathrm{mM}$ 2-oxoglutarate. 
potentiostat (Hokuto Electronics Ltd., Tokyo) and a recorder (Toa Electronics Ltd., Tokyo). In B, the flow stream was split after sample injection and rejoined before GLDH reactor by two T-connectors, a delay coil (Teflon tube, $1.0 \mathrm{~mm}$ i.d., $1.2 \mathrm{~m}$ long) was set at the channel containing the urease reactor (urea channel). The components of the manifolds were connected with silicon and Teflon tubings and the distances between the injector, the enzyme reactors and the flow cell were kept as short as possible in order to minimize sample dispersion. The working solution was propelled into the systems at a rate of $1.6 \mathrm{ml} / \mathrm{min}$. The samples $(10$ or $50 \mu 1)$ were injected with a microsyringe.

\section{Results and Discussion}

\section{Enzymatics}

The support for enzyme immobilization was CPG. Coupling of the enzymes on propylamine-CPG pretreated with glutaraldehyde gave high activity yields for CRDI, urease and GLOD (Table 1). This method, however, showed extremely low residual activity in the case of GLDH. Several methods were investigated for the immobilization of GLDH. The best result was obtained by coupling the enzyme onto succinate-CPG activated with EDC, a water soluble carbodiimide, through peptide bound.

The values of the Michaelis-Menten constants $\left(K_{\mathrm{m}}\right)$ for both native and immobilized enzymes are also shown in Table 1. While the $K_{\mathrm{m}}$ values of urease, CRDI and GLOD were enlarged by immobilization for 1.5-3 times, immobilized GLDH showed a much lower $K_{\mathrm{m}}$ for ammonia than the native one. In an FIA system based on coupled enzyme reactions, since the concentration of the analyte is very low, the total analyte conversion efficiency is limited by the reaction catalyzed by the enzyme with the highest $K_{\mathrm{m}}$ value. Thus, a large decrease of $K_{\mathrm{m}}$ for ammonia of GLDH is of great significance with regard to substrate conversion, since a high conversion efficiency with a small quantity of immobilized enzymes results in a high assay sensitivity.

\section{Choice of working solution}

Urease from thermophilic Bacillus sp. was used in this study, though the activity of this enzyme (4.5 units/mg powder) is much lower than that from jack bean (100 units/mg powder), which is exclusively used for almost all analytical purposes. The advantage of using this enzyme in the present study is that its pH optimum is located at the alkaline side, and it shows a much lower $K_{\mathrm{m}}$ (Table) than does urease from jack bean $(10.5 \mathrm{mM}) .^{13}$

The $\mathrm{pH}$ dependences of immobilized enzymes are shown in Fig. 2. The four enzymes gave a common $\mathrm{pH}$ optimum near 8.0. This made it possible to use a single working solution to run the FIA system, thus eliminating the complicated procedure to regulate the working $\mathrm{pH}$ for each enzyme. To determine the optimum concentration of NADH, the conversion efficiency of ammonia to glutamate was investigated using the singlechannel system (Fig. 1A, injection volume, $50 \mu \mathrm{l}$ ). The substrate concentration was $2.0 \mathrm{mM}$, which was the upper limit of the linear response. The conversion was near to complete for a NADH concentration larger than $0.25 \mathrm{mM}$. Thus, $0.3 \mathrm{mM}$ of NADH was used in the working solution. This concentration may be insufficient for a higher substrate concentration. At a higher concentration area, however, the limit step of the system response to creatinine is the GLOD-catalyzed oxidation of glutamate, due to the restriction of the dissolved oxygen level (since the oxygen concentration in airsaturated pure water is about $0.25 \mathrm{mM}$ at $25^{\circ} \mathrm{C}$, the dissolved oxygen level in a working solution should be

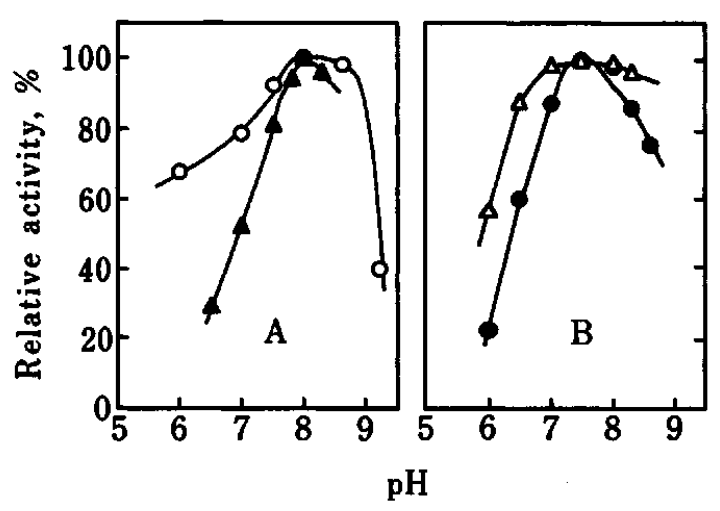

Fig. 2 pH dependences of immobilized enzymes: $A$, urease $(\Delta)$ and CRDI $(O) ; B, G L D H(\odot)$ and GLOD $(\Delta)$.

Table 1 Results of enzyme immobilization and the kinetics of native and immobilized enzymes

\begin{tabular}{llcccc}
\hline Enzyme & Immobilization method & $\begin{array}{c}\text { Activity bound } \\
\text { (units/g dry CPG) }\end{array}$ & Residual activityb, $\%$ & \multicolumn{2}{c}{$K_{\mathrm{m}} / \mathrm{mM}$} \\
\hline CRDI & GR & 106 & 101 & Native & Immobilized \\
Urease & GR & 259 & 93.9 & 1.3 & 3.6 \\
GLDH & GR & 8.5 & 3.1 & $30.3^{\mathrm{c}}$ & 0.80 \\
& GDC & 120 & 25.8 & & N.T. $^{\mathrm{d}}$ \\
GLOD & GR & 97.7 & 82.3 & $0.22^{\mathrm{c}}$ & $0.35^{\mathrm{e}}$ \\
\hline
\end{tabular}

a. GR, on glutaraldehyde-pretreated propylamine-CPG, EDC, on succinate-CPG activated with 1-ethyl-3-(3-dimethylaminopropyl)carbodiimide. b. Residual activity $(\%)=$ (specific activity of immobilized enzyme/specific activity of native enzyme) $\times 100$. c. For ammonia. d. Not determined. e. For glutamate. 
lower than $0.25 \mathrm{mM}$ ). CLDH was more stable in a phosphate buffer than in a Tris- $\mathrm{HCl}$ or borate buffer (data not shown), though this was not the case for other enzymes. Therefore, a $67 \mathrm{mM}$ phosphate buffer (pH 8.0) containing $0.1 \mathrm{mM}$ EDTA, $0.3 \mathrm{mM} \mathrm{NADH}$ and $5 \mathrm{mM}$ 2-oxoglutarate was used as the working solution. EDTA was added as an enzyme stabilizer.

\section{Determination of urea and creatinine with the single channel FIA system}

Enzyme reactors were sequentially aligned in the order of urease or CRDI, GLDH and GLOD, as shown in Fig. 1A. Standard samples of urea or creatinine were manually injected through the injection port with a microsyringe. Figure 3 shows the calibration curves for

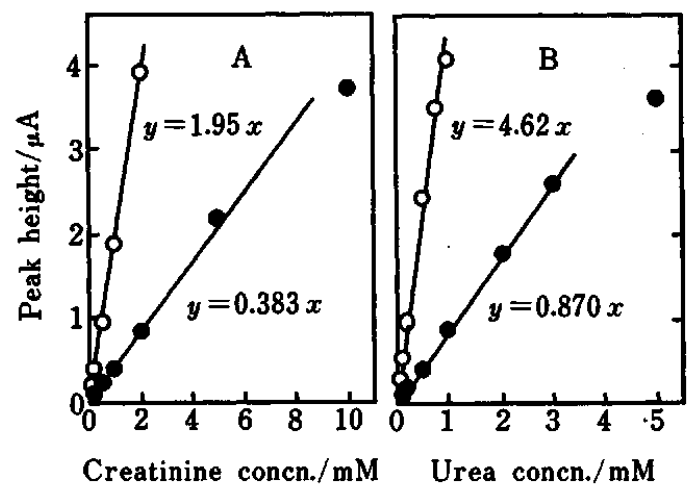

Fig. 3 Calibration curves of the single channel FIA system for (A) creatinine and (B) urea. Injection volume of samples: (O) $10 \mu \mathrm{l}$, (O) $50 \mu \mathrm{l}$. The calibration equations are indicated as $y$ being the peak height and $x$ being the concentration. See Fig. 1 for other experimental conditions. urea (A) and creatinine (B). The linear calibration range of the system depended on the injection volume of the samples. For urea, the calibration range was 0.1 $3 \mathrm{mM}$ for $10 \mu \mathrm{l}$ injection and $0.02-0.75 \mathrm{mM}$ for $50 \mu \mathrm{l}$ injection. For creatinine the corresponding range was $0.2-5 \mathrm{mM}$ and $0.05-2 \mathrm{mM}$. The slopes of calibration curves for $50 \mu$ l injection were approximately 5-times lager than those for $10 \mu$ injection, proportional to the ratio of injection volumes. The sensitivity for urea was about two-fold higher than that for creatinine, corresponding to the fact that the hydrolysis of one molecule of urea produces two molecules of ammonia. The response time for both compounds was within $30 \mathrm{~s}$.

\section{Delay coil and flow distribution}

The principle for the simultaneous determination of two components, as shown in Fig. $1 \mathrm{~B}$, is the split, and subsequent confluence, of the flow stream. Doublepeak recording resulted from a delay coil set at the urea channel. The first peak represents the response to creatinine and the second to urea.

Among the various factors affecting the separation and relative heights of the two peaks, the delay coil (length, inner diameter and material) and the distribution of flow over the two channels are the most important. To determine the optimum length of the delay coil, the responses of the two channel system to ammonia under different lengths of delay coils were investigated (Fig. 4A-D). The flow rates indicated in the figure were measured under an open state behind of CRDI and urease reactors, which might not represent the real rate when the GLDH/GLOD reactor is connected, but do give a parameter of flow distribution over the two channels. The flow distribution was controlled by regulating the length of the urease or the CRDI reactor. When freshly prepared columns were used, the dis-

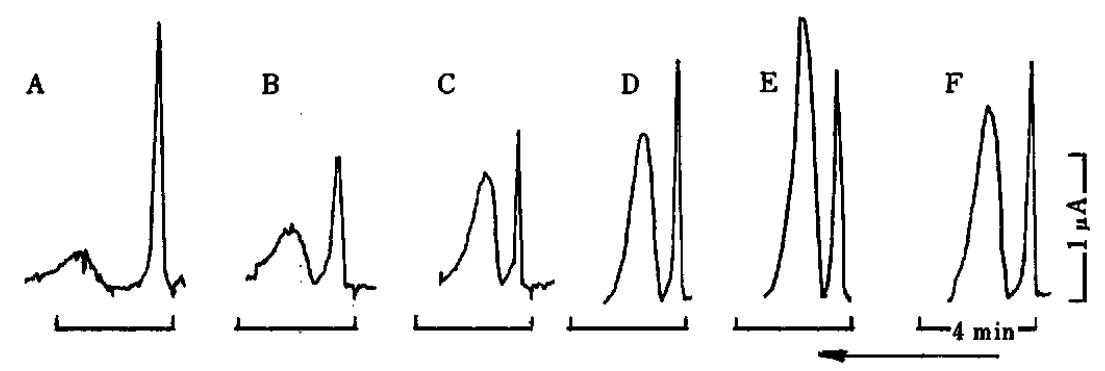

Fig. 4 Effects of the delay coil and flow distribution on the response of the two-channel FIA system. A-D, responses to $2.0 \mathrm{mM}$ ammonium chloride; $E$ and $F$, responses to mixed sample of creatinine and urea with an equal concentration of $2.0 \mathrm{mM}$. Lengths of the delay coil (m) and flow rates (ml/ min, measured at open state before GLDH column) through the two channels are (delay coil, flow rate (1st channel), flow rate (2nd channel)): $\mathbf{A}(3.0,0.82$, $0.80), \mathrm{B}(2.0,0.79,0.83), \mathrm{C}(1.5,0.81,0.78), \mathrm{D}(1.2,0.81,0.79), \mathrm{E}(1.2,0.81$, $0.79), E(1.2,0.85,0.74)$. The respective lengths of the urease column were $15 \mathrm{~mm}$ in $A$ and $B ; 16.5 \mathrm{~mm}$ in $\mathrm{C}, \mathrm{D}$, and $\mathrm{E}$; and $17 \mathrm{~mm}$ in $\mathrm{F}$. The chart direction is indicated by an arrow (right to left). See Fig. 1 for the other conditions. 


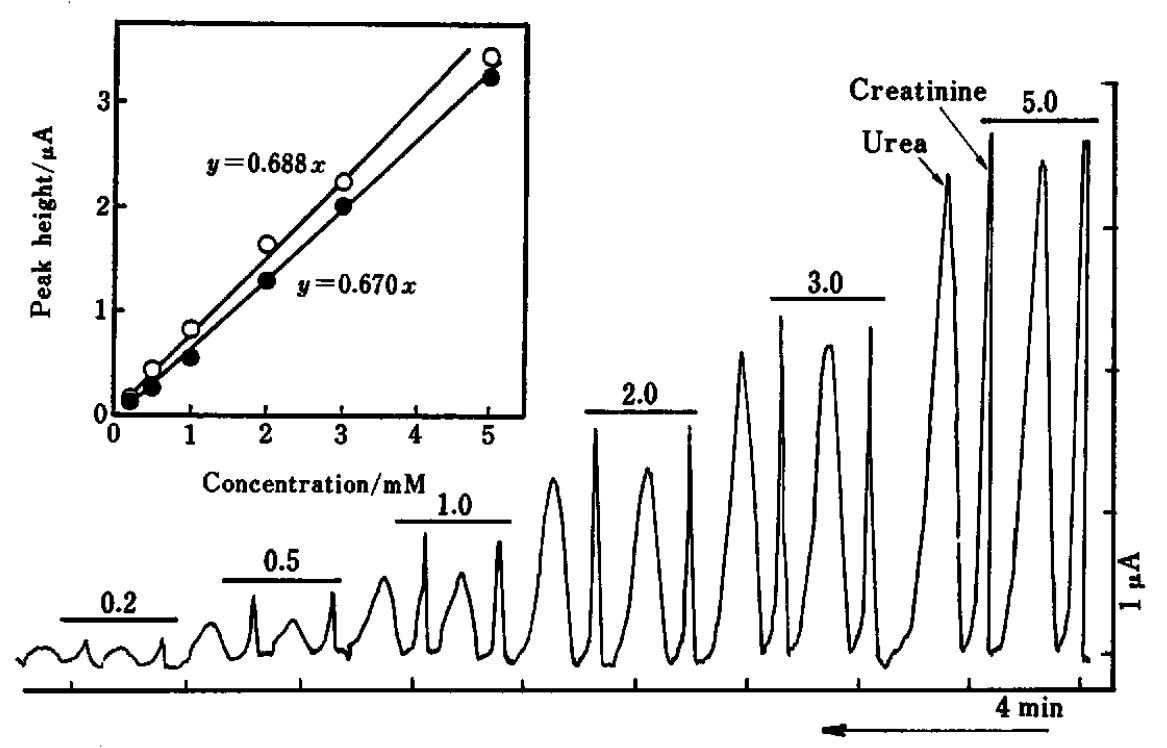

Fig. 5 Recorder responses of the two channel FIA system to standard samples of creatinine and urea. The concentrations are shown in $\mathrm{mM}$. The chart direction is indicated by an arrow. The injection volume of samples was $50 \mu \mathrm{l}$. Inset: the corresponding calibration curves for creatinine $(O)$ and urea (O). The calibration equations are indicated as $y$ being the peak height and $x$ being the concentration. See Fig. 1 for other conditions.

tribution of flow was not stable within the initial $1-2 \mathrm{~h}$; however, good stability was constantly obtained after the system was previously run with phosphate buffer for $2-3 \mathrm{~h}$. Obviously, the height of second peak increased with decreasing length of the delay coil. At the same time, the second peak became sharper and the total response time for one injection was shortened from $4.5 \mathrm{~min}$ to $2.5 \mathrm{~min}$.

When a mixed solution of creatinine and urea with an equal concentration was injected (Fig. 4E), the second peak of urea became higher than the first peak of creatinine. The flow rate through the urease channel was thus slightly reduced by increasing the length of the urease reactor. As a result, the second peak became greatly lower (Fig. 4F). Therefore, the relative heights of the two peaks were also affected by the flow distribution. On the other hand, the relative assay sensitivity of the two channels could be regulated by simply controlling the flow distribution. The following experiments were performed under the same condition as given in Fig. 4F.

\section{Simultaneous determination of creatinine and urea with two channel FIA system}

Figure 5 shows the recorder response to standard samples of creatinine and urea of the two-channel FIA system, and the corresponding calibration curves (inset). Linear relationships between peak heights and the concentrations were obtained up to $5 \mathrm{mM}$ for both creatinine and urea (injection volume, $50 \mu \mathrm{l}$ ). As shown in the figure, the reproducibility was good (the relative standard deviation for 12 repeated run was less than $5 \%$ for either of the two peaks) and one run could be finished within $3 \mathrm{~min}$. The operational stability of the system depends on the lifetime of the enzyme reactors. When stored in a phosphate buffer $(\mathrm{pH} 7.5)$ at $4^{\circ} \mathrm{C}$, the most unstable GLDH retained $90 \%$ of its original activity over one month and $70 \%$ over a year. The response of the FIA system began to decrease after operation for about 3 weeks (approx. 1400 times of injection). However, linear calibrations could still be obtained over 6 months' occasional operation.

The lower detection limits for both creatinine and urea were $0.2 \mathrm{mM}$. The sensitivity fairly decreased when compared with that of the single-channel system (Fig. 2). This decline of sensitivity is attributed to a dilution effect of the split and subsequent confluence of the flow stream. If we consider the split of the sample zone as being a kind of diluting procedure, the dilution factor will be sequared at the confluence point. For example, the total dilution factors in the present system for creatinine and urea should be about $(1.79 / 0.85)^{2}=3.5$ and $(1.79 / 0.74)^{2}=4.6$, respectively.

If necessary, however, the sensitivity could be enhanced by increasing the injection volume of the sample (ref. Fig. 3). Rather, we can take advantage of the dilution effect of the split and confluence of flow to regulate the dynamic range for each channel. This is especially significant in an assay of samples containing greatly different amounts of analytes. For instance, the concentration of urea is more than 10-times higher than that of creatinine in normal biological samples, such as urine and serum. Since two moles of ammonia are formed from one mole of urea, this difference is doubled 
to larger than 20 fold. It is almost impossible to simultaneously assay the two components in such samples with a system of equally sensitive to them. Since the dynamic range of amperometric sensors is usually small (upper limit/lower limit $<100$ ), a 10-fold difference between the dynamic ranges for urea and creatinine should be made. Improvements of the twochannel FIA system, including differentiation of the dynamic ranges for creatinine and urea, and the overcoming of interfering endogenous ammonia and glutamate, are under way; the simultaneous determination of creatinine and urea in human urine samples will be reported in near future.

This work was supported in part by a Grant-in-Aid for Scientific Research from the Ministry of Education, Science and Culture, Japan.

\section{References}

1. I. Karube, H. Matsuoka, S. Suzuki, E. Watanabe and K. Toyama, J. Agric. Food Chem., 32, 314 (1984).

2. S. Peguin, P. R. Coulet and G. Bardeletti, Anal. Chim.
Acta, 222, 83 (1989).

3. M. Valcarcel, M. D. Luque de Castro, F. Lazaro and A. Rios, Anal. Chim. Acta, 216, 275 (1989).

4. T. Murachi and M. Tabata, in "Baiosensa (Biosensor, in Japanese)”, ed. S. Suzuki, p. 176, Kodansha, Tokyo, 1984.

5. I. Karube, T. Okada and S. Suzuki, Anal. Chem., 53, 1852 (1981).

6. T. Okada, I. Karube and S. Suzuki, Eur. J. Appl. Microbiol. Biotechnol., 14, 149 (1982).

7. I. Kubo, I. Karube and S. Suzuki, Anal. Chim. Acta, 151, 371 (1983).

8. T. Murachi and M. Tabata, Biotechnol. Appl. Biochem., 9, 303 (1987).

9. H. H. Weetall, Methods Enzymol., 44, 134 (1976).

10. I. C. Cho and H. Swaisgood, Biochim. Biophys. Acta, 334, 243 (1974).

11. H. Kaltwasser and H. G. Schlegel, Anal. Biochem., 16, 132 (1966).

12. A. Bohmer, A. Muller, M. Passarge, P. Liebs, H. Honeck and H.-G. Muller, Eur. J. Biochem., 182, 327 (1989).

13. F. J. Reithel, The Enzymes (3rd ed.), 4, 1 (1971).

(Received July 21, 1992)

(Accepted September 24, 1992) 